\title{
National microbiological surveillance of the susceptibility of gonococcal isolates to antimicrobial agents
}

\author{
JO-ANNE R DILLON
}

$\mathrm{T}$ HE INCIDENCE OF GONORRHEA HAS BEEN DECLINING IN Canada and other industrialized countries; in $1981,56,330$ cases of gonorrhea were reported in Canada compared with 13,822 cases in 1990 (1). The reasons for this decline are unknown; however, educational programs for the primary prevention of acquired immune deficiency syndrome as well as effective therapy may have played a role. Persons aged 15 to 29 years accounted for more than $75 \%$ of all reported cases of gonorrhea with twice as many female cases in the 'under 20' age group. Females with gonorrhea are at increased risk for serious complications, such as pelvic inflammatory disease (PID) which, in turn, may lead to sequelae such as infertility and ectopic pregnancy. Untreated or ineffectively treated gonococcal infections are a significant cause of PID.

Despite the decrease in the number of cases of gonorrhea, the prevalence of isolates resistant to antimicrobial agents used for treatment has steadily increased in Canada. This resistance is caused by either chromosomal mutations or plasmids conferring antibiotic resistance. A number of chromosomal loci which produce phenotypes with reduced susceptibility to antimicrobial agents have been described (2). Some chromosomal mutations are characterized by simultaneous resistance to other unrelated antimicrobial agents and some act in concert to produce step-wise. additive increases in resistance. Decreased antimicrobial susceptibility in gonococci caused by chromosomal

National Laboratory for Sexually Transmitted Diseases, Laboratory Centre for Disease Control, Ottawa, Ontario

Correspondence and reprints: JR Dillon, Health and Welfare Canada, National Laboratory for Sexually Transmitted Diseases, Laboratory Centre for Disease Control, Ottawa, Ontario K1A OL2 mutation (as well as plasmid-mediated mechanisms) leads to increased frequencies of treatment failure, requiring the periodic revision of treatment guidelines which reflect the specific antimicrobial susceptibilities of a country or region. Chromosomal resistance is defined as a minimum inhibitory concentration (MIC) of at least $2 \mathrm{mg} / \mathrm{L}$ for penicillin, tetracycline and erythromycin and an MIC of at least $128 \mathrm{mg} / \mathrm{L}$ for spectinomycin (3), Breakpoints have not been established for antimicrobials such as ceftriaxone. For quinolone antibiotics such as ciprofloxacin, an MIC of 0.25 to 0.5 $\mathrm{mg} / \mathrm{L}$ has been considered resistant (4). In Canada, although plasmid-mediated resistance to either penicillin or tetracycline has replaced chromosomal resistance as the major mechanism of resistance in many regions, the development of chromosomal resistance remains an important potential cause of treatment failure, particularly to newer antimicrobials such as the quinolones, cefixime and ceftriaxone. For example, the 1988-89 national antimicrobial susceptibility surveillance study undertaken by our laboratory indicated that $1.4 \%$ of 3031 gonococcal isolates were chromosomally resistant to penicillin and $2.6 \%$ were resistant to tetracycline. Isolates with chromosomally-mediated penicillin resistance were also resistant to one to three unrelated antibiotics such as tetracycline, erythromycin and cefoxitin. The total burden of chromosomal resistance observed in the study, including all combinations of antibiotic resistance tested and resistance to erythromycin, was $15.5 \%$ of the isolates.

In many areas of Canada, the most clinically important mechanism of resistance is plasmid-mediated resistance to either penicillin or tetracycline. The first penicillinase-producing isolates of Neisseria gonorrhoeae (PPNG) in Canada were isolated in 1976 (2). 
PPNG strains carry one of a family of related plasmids that produce the TEM-1 type, beta-lactamase. In addition to carrying an enzyme that destroys penicillin, PPNG isolates may also exhibit chromosomal resistance to antibiotics such as tetracycline and, in rare instances, spectinomycin or quinolone antimicrobials. The production of a beta-lactamase is indicative of a PPNG isolate; these strains generally have MICs greater than $16 \mathrm{mg} / \mathrm{L}$; however, in rare cases, isolates with lower MICs may produce beat-lactamase. All beta-lactamase-producing gonococci, irrespective of MICs, are considered to be clinically resistant to penicillins.

In 1986, the first $N$ gonorrhoeae isolates from Canada with plasmid-mediated resistance to tetracycline (TRNG) were reported in British Columbia (5). TRNG isolates acquired the tet $M$ determinant within the gonococcal conjugative plasmid (6). TRNG isolates are presumptively identified based on their antimicrobial susceptibility to tetracycline; isolates with an MIC of 2 to $8 \mathrm{mg} / \mathrm{L}$ are considered to be chromosomally resistant and isolates with an MIC of at least $16 \mathrm{mg} / \mathrm{L}$ are considered to carry plasmid-mediated resistance to tetracycline (3). Plasmid-mediated resistance is identified only definitely through the use of tetM-specific diagnostics such as DNA probing or gene amplification strategies such as the polymerase chain reaction.

Because of the clinical importance of antibiotic resistant gonococci, the National Laboratory for Sexually Transmitted Diseases (NLSTD) has undertaken national collaborations with provincial public health laboratories and other centres to determine the prevalence of antibiotic resistance in gonococcal isolates from Canadian centres. Since the isolation of the first PPNG isolate in 1976, we have requested that all gonococcal isolates with either plasmid-mediated or chromosomal resistance to antimicrobial agents be sent to the NLSTD for national documentation, confirmation of and extension of antimicrobial susceptibility profiles and strain typing (plasmid content analysis; auxotype, ie, nutritional requirement determination; and serotyping with a panel of monoclonal antibodies). In addition, periodic retrospective (1973-74, 1978-79) or prospective (198889, 1991-92) national collaborative studies have been undertaken to determine the antimicrobial susceptibility of gonococcal isolates from across Canada which do not carry plasmid-mediated resistance, to evaluate temporal and geographic changes in susceptibility and to determine the molecular epidemiology of gonococcal isolates across Canada. In turn, the data are used to develop national recommendations for the treatment of gonorrhea as well as to develop models of gonococcal strain transmission that may impact on epidemiological practices. For example, from March 1, 1988 to March 31, 1989 we conducted a national collaborative study to determine the clinical and biological factors influencing gonococcal infections and treatment in children and adults in Canada. This was the first study in Canada in which consecutive isolates of $\mathrm{N}$ gonorrhoeae were prospectively collected from 26 participating centres across Canada; 19\% of all reported cases of gonorrhea were tested. Notable results of the study included the first national data on the prevalence of isolates with chromosomal resistance, and documentation of reduced susceptibility of isolates to certain antimicrobial agents (eg, the MIC9os of isolates to spectinomycin doubled since 1978 from 8 to $16 \mathrm{mg} / \mathrm{L}$ and the MICs of isolates to ceftriaxone exhibited a biphasic distribution with the less susceptible isolates correlating with resistant strain types). In addition, significant geographic differences in susceptibility and strain types were documented, with isolates from the Atlantic provinces and some areas of the Northwest Territories being more susceptible to penicillin and tetracycline than isolates from other regions of Canada. Comparison of these data with previous national studies indicated that the susceptibility to penicillin of isolates which do not carry plasmid-mediated resistance to penicillin or tetracycline decreased from $45 \%$ of 732 gonococcal isolates in 1973 to $19.2 \%$ of 608 isolates in 1991. These shifts in antimicrobial susceptibility have also been reflected in shifts in the predominant strain type as determined by auxo/serotyping methods.

Gonococcal isolates which inactivate penicillin PPNG represented $9.6 \%$ of all reported cases of gonorrhea in 1990 compared with 5\% in 1985. The distribution of PPNG in Canada has shifted from a sporadic distribution across the country to clustering in urban areas, for example Metro Toronto. In 1991, 64.8\% (827 of 1277 isolates) of all PPNG submitted to the NLSTD were recovered from the Metro Toronto area. In 1988, 1.7\% (10 of 591) of PPNG isolates also carried plasmidmediated resistance to tetracycline (PP/TRNG); by 1991, the number of PP/TRNG increased to 568 isolates with $74 \%$ of these isolates from the Toronto area. The number of gonococcal isolates with plasmidmediated resistance to tetracycline has also increased from six isolates in 1986 to 890 isolates in 1991. While the incidence of TRNG is still under $1 \%$ of reported cases of gonococci, their steady increase in prevalence is reminiscent of the steady increase of PPNG isolates over a decade ago.

Because the prevalence of resistant isolates changes temporally and geographically, the efficacy of antimicrobials used clinically must be monitored continually. For this reason the national antimicrobial susceptibility monitoring study of 1988-89 has been re-initiated and the network of collaborating centres has been expanded. The sample of gonococci collected is proportional to the number of gonococci reported from a given area. In addition, the NLSTD will continue to collect and type all PPNG and TRNG isolated in Canada. In order to encourage local laboratories to monitor the susceptibility of the gonococcal isolates in their regions, a national antimicrobial susceptibility 
quality control program has been initiated with eight centres participating. In the program, standard methods for testing the antimicrobial susceptibility of $N$ gonorrhoeae isolates have been implemented following the methods recommended by the National Committee for Clinical Laboratory Standards (3). The NLSTD provides quality assurance for specific lots of GC agar base, providing these and reference strains to participants. In addition to the microbiological studies, the NLSTD is collaborating with the Division of STD Control, Bureau of Communicable Disease Epidemiology, to collect retrospective case history data for data linkage. Findings regarding significant national trends are communicated to various groups and study participants as well as to appropriate authorities on an on-going, timely basis. Study centres are encouraged to publish data from their regions and forums are provided for discussing the impact of regional and national trends as well as to review and develop national recommendations for the treatment of gonorrhea.

The impact of the 1988-89 study, together with on-going surveillance of antibiotic-resistant gonococci, was underlined in March 1991 when a national working group convened in Ottawa to review national guidelines for the diagnosis and treatment of STDs. At that time, significant changes to the recommended treatments for gonorrhea were made. Because of the high overall prevalence of isolates resistant to penicillin/ampicillin and tetracycline, these agents could no longer be recommended for the treatment of uncomplicated gonococcal infections (either singly or in combination) unless active monitoring for resistance to penicillin was undertaken, if the percentage of penicillin-resistant isolates was under $3 \%$ and if the infection was acquired in the same geographic area. Ceftriaxone (250 mg intramuscular in a single dose) was recommended as the preferred therapy for treatment of uncomplicated gonococcal in- fections in adolescents and adults (7). Several oral regimens were also recommended: cefixime $(800 \mathrm{mg}$ in a single dose), ciprofloxacin (500 $\mathrm{mg}$ in a single dose) and ofloxacin (400 $\mathrm{mg}$ orally in a single dose). Spectinomycin ( 2 gm intramuscular in a single dose) continues to be an effective alternative therapy. Because the risk of having simultaneous chlamydia infections is high, treatment for gonococcal infections must be accompanied by treatment for chlamydial infections (ie, doxycycline/tetracycline). Information regarding treatment of other gonococcal infections or for treating gonococcal infections in children can be obtained from the Canadian guidelines for the prevention, diagnosis, management and treatment of STDs in neonates. children, adolescents and adults (7).

\section{REFERENCES}

1. Health and Welfare Canada. Notifiable diseases annual summary. 1991:1-145.

2. Dillon JR, Yeung K-H. $\beta$-lactamase plasmids and chromosomally mediated antibiotic resistance in pathogenic Neisseria species. Clin Microbial Rev 1989:2S: 125-33.

3. National Committee for Clinical Laboratory Standards. Methods for dilution antimicrobial susceptibility tests for bacteria that grow aerobically. Document M7-A2. 1990:10:1-25.

4. Jephcott AE, Turner A. Ciprofloxacin resistance in gonococci. Lancet 1990;335:165.

5. Shaw CE, Chan DGW, Byrne SK, Black WA, Bowie WR. Tetracycline-resistant Neisseria gonorrhoeae (TPNG) British Columbia. Can Dis Wkly Rep 1986;12:101.

6. Morse SA, Johnson SR, Biddle JW, Roberts MC High-level tetracycline-resistance in Neisseria gonorrhoeae is result of acquisition of streptococcal TetM determinant. Antimicrob Agents Chemother 1986;30;664-70.

7. Health and Welfare Canada, Canadian guidelines for the prevention, diagnosis, management and treatment of sexually transmitted diseases in neonates, children, adolescents and adults. Can Commun Dis Rep 1992:18S1:1-213. 


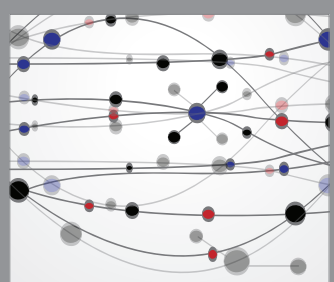

The Scientific World Journal
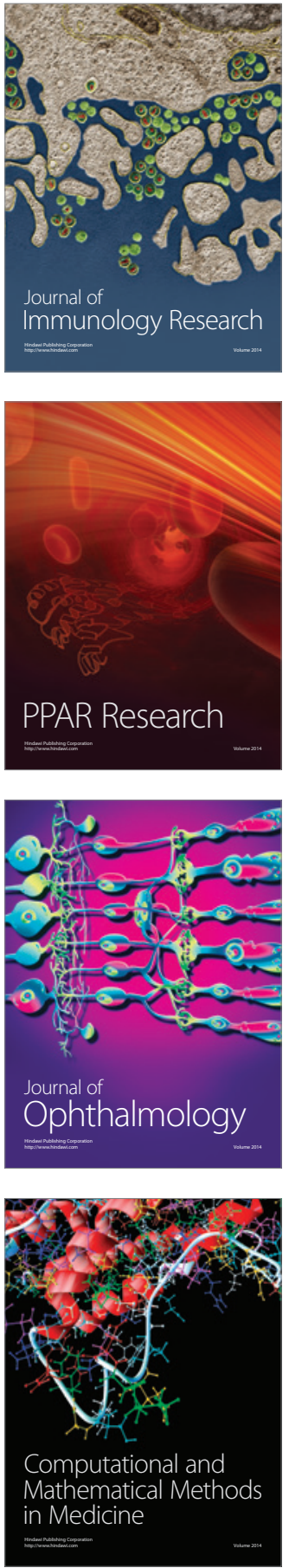

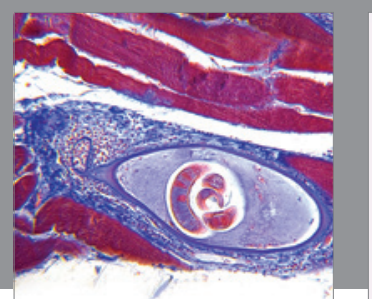

Gastroenterology Research and Practice

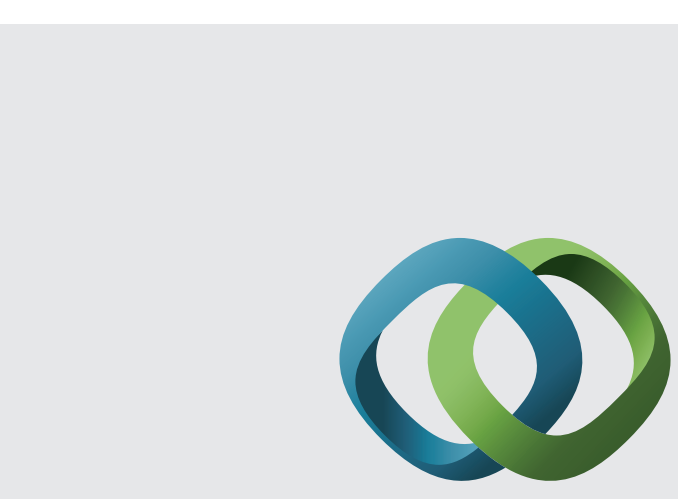

\section{Hindawi}

Submit your manuscripts at

http://www.hindawi.com
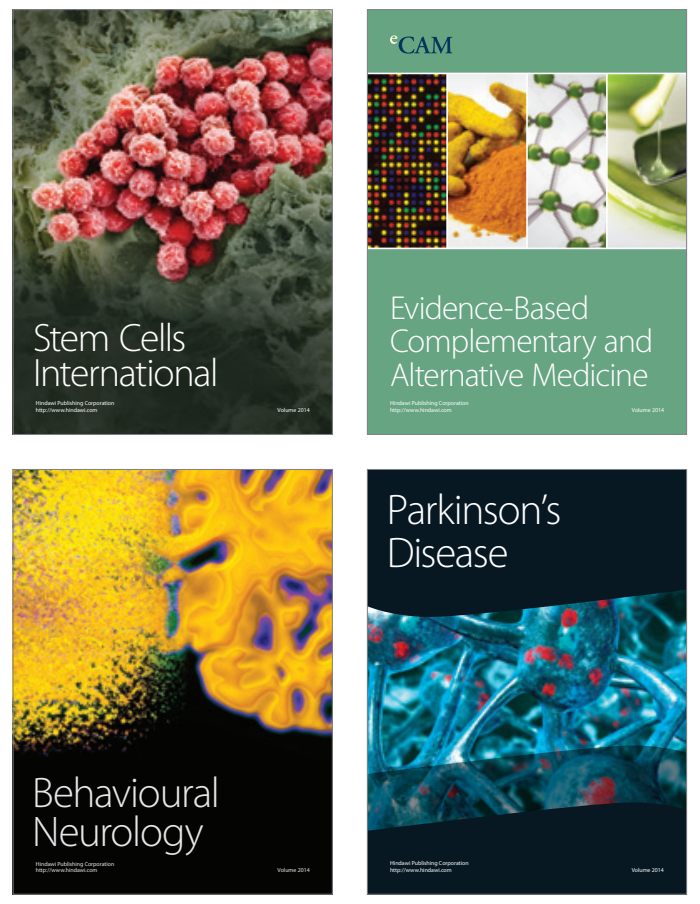
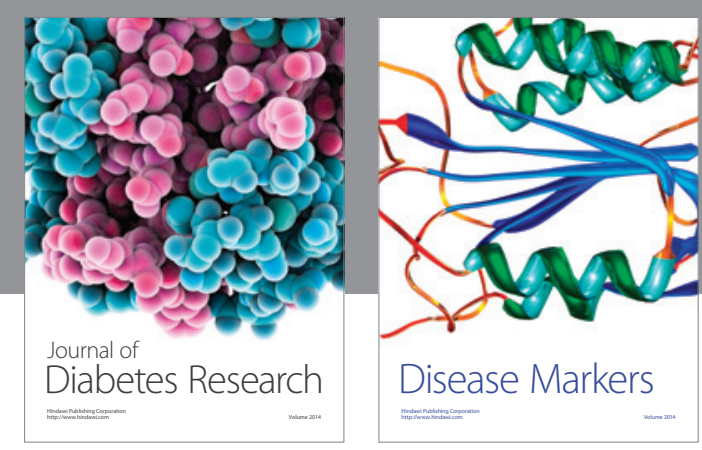

Disease Markers
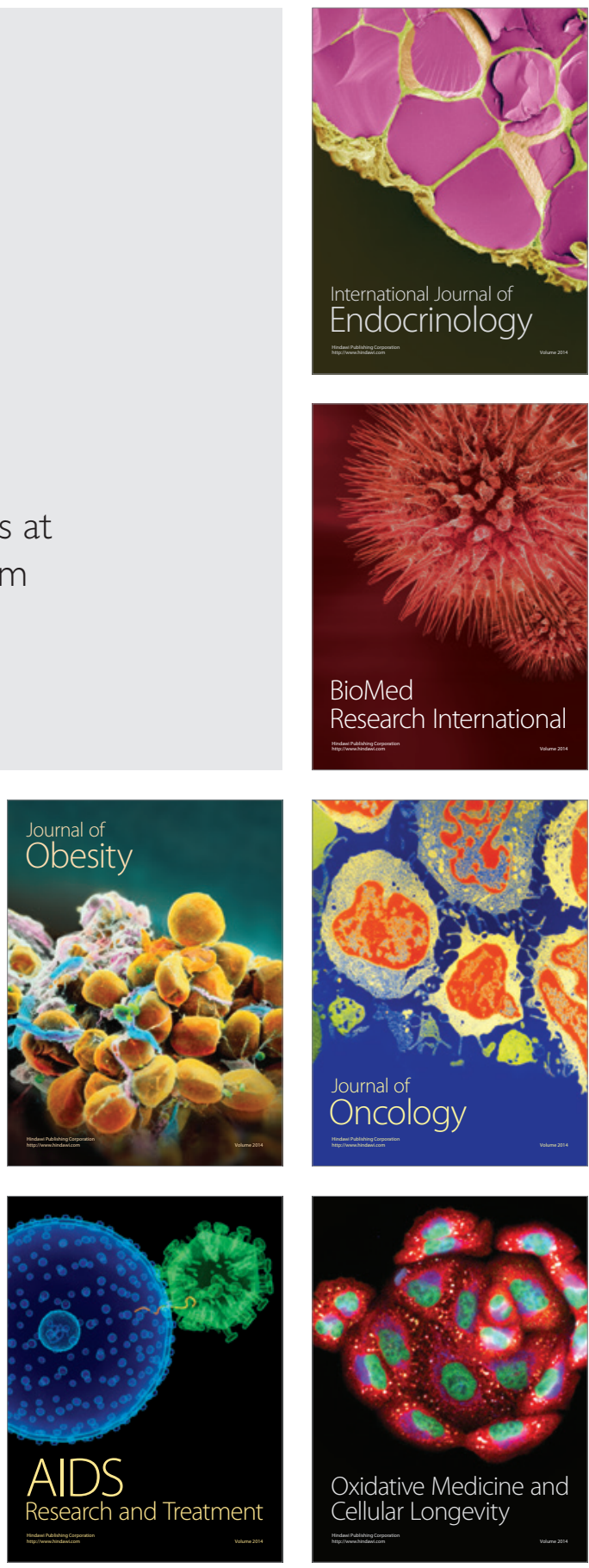